\title{
(6) OPEN ACCESS \\ Self-reported preparedness for clinical work has increased among recent cohorts of UK-trained first-year doctors
}

\author{
Shelly Lachish, Michael I Goldacre, Trevor Lambert
}

\begin{abstract}
- Additional material is published online only. To view please visit the journal online (http://dx.doi.org/10.1136/ postgradmedj-2015-133858).

UK Medical Careers Research Group, Nuffield Department of Population Health, University of Oxford, Oxford, UK
\end{abstract}

\section{Correspondence to}

Shelly Lachish, Unit of HealthCare Epidemiology, Nuffield Department of Population Health, University of Oxford, Old Road Campus, Old Road, Oxford OX3 7LF, UK: shelly.lachish@dph.ox.ac.uk

Received 19 November 2015 Revised 7 January 2016 Accepted 2 February 2016 Published Online First 22 February 2016

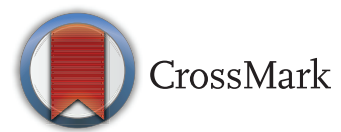

To cite: Lachish $S$, Goldacre MJ, Lambert T. Postgrad Med J 2016:92:460-465.
ABSTRACT

Introduction UK medical schools have made considerable efforts to ensure that graduates are well prepared for their first year of clinical work. We report the views of two recent cohorts of UK-trained doctors 1 year after graduation about whether their medical school prepared them well, and compare responses with earlier cohorts.

Methods We surveyed doctors who qualified in 2011 and 2012 from all UK medical schools. We obtained their responses to the statement 'My experience at medical school prepared me well for the jobs I have undertaken so far' on a 5-point scale from 'Strongly Agree' to 'Strongly Disagree'. Responses were compared with those of the UK graduates of 1999, 2000, 2002, 2005, 2008 and 2009, surveyed in the same way 1 year after graduation.

Results The percentage of doctors who either 'Agreed' or 'Strongly Agreed' that they were well prepared doubled from 35\% in 1999 to $70 \%$ in 2012, while the percentage who 'Strongly Agreed' with the statement increased fourfold. Perceptions of being well prepared have increased in graduates from almost every medical school. Variation between medical schools in selfreported preparedness of their graduates has decreased in recent cohorts. However, some large differences between medical schools remain. Significant differences in perceived preparedness remain between white and non-white doctors, but have diminished between men and women.

Conclusions Our work contributes to growing evidence suggesting that changes to medical education in the UK are producing doctors who feel well prepared for the challenges of being a doctor, though further improvements could be made.

\section{INTRODUCTION}

The primary tasks of medical schools are to provide students with a firm basis for the development of a working lifetime in medical practice and to ensure that their graduates are well equipped with the necessary knowledge and skills for the first stage of their professional career as junior doctors. Numerous studies, however, have shown that medical graduates often feel ill-prepared for the demands of their new job. ${ }^{1-4}$ In the first 2 years of clinical practice (termed the foundation years in the UK), foundation doctors work under the close supervision of more senior doctors. Nevertheless, many graduates find the transition from student to junior doctor to be highly stressful. ${ }^{5} 6$

Consequently, medical education and training has undergone extensive reforms in many countries in recent years. Over the past 15 years, UK medical schools have restructured clinical courses to better deliver the directives for learning and competencies outlined in the General Medical Council's (GMC) 'Tomorrow's Doctors' reports. ${ }^{7} 8$ Changes have included scenario-based and problem-based approaches as well as traditional lecture-style courses and bedside teaching; the use of 'student-led' learning under appropriate supervision; a focus on teaching core curricula to small groups of students; and the integration of clinical and non-clinical learning across disciplines. ${ }^{9}$ In recent years, the GMC has further updated its standards for excellence in medical education. ${ }^{10}{ }^{11}$ In particular, the most recent update of Tomorrow's Doctors places considerable emphasis on clinical assistantships and 'shadowing' (students working closely alongside qualified doctors undertaking their routine clinical duties) in the final year of medical school. ${ }^{10}$ Accordingly, medical schools have continued to modify and adapt their curricula and educational programmes to meet these standards. ${ }^{12}$

One means of assessing whether such changes undertaken by medical schools are succeeding in their task of producing better-equipped graduates is to seek the views of the graduates themselves regarding whether their medical school prepared them well for work. In our previous research on medical careers, we reported a moderate, but variable, increase in self-assessed preparedness for work from 1999 to 2009 expressed by junior doctors towards the end of their first postgraduate year and also showed persistent and sizeable differences between medical schools and demographic subgroups. ${ }^{2} 13-15$

In this study, we update our previous findings on self-reported preparedness in junior doctors with data obtained from the graduates of 2011 and 2012, to evaluate the extent to which continuing reform of medical education is associated with improvements in self-reported preparedness for practice.

\section{METHODS}

We contacted graduates from all UK medical schools towards the end of their first foundation year of postgraduate training (the F1 year) via addresses obtained from GMC registrations. Registration with the GMC is mandatory in order to practise medicine in the UK. Graduates of 2012 were sent questionnaires by mail in mid-2013 and were also contacted by email with the option to complete a web-based version of the survey. Up to 
four reminders were sent to non-respondents. Graduates of 2011 were surveyed a year earlier in mid-2012 via a trial 'web-only' approach and were only contacted via email and invited to complete the web-based survey.

Our surveys were multipurpose and included questions about future career intentions and factors affecting them, experiences of the F1 year and views on training and work. Among other themes, we invited doctors to respond to the statement " $\mathrm{My}$ experience at medical school prepared me well for the jobs I have undertaken so far" on a five-point scale from 'Strongly Agree' to 'Strongly Disagree'. Those who did not 'Strongly Agree' or 'Agree' to the statement were asked to indicate whether 'lack of preparation was a serious, medium-sized, or minor problem' for them, and to indicate in which of five areas of work they felt unprepared ('clinical knowledge', 'clinical procedures', 'administrative tasks', 'interpersonal skills', 'physical/ emotional/mental demands'). Some respondents who had agreed with the preparedness statement overall, nevertheless, indicated that they did not feel well prepared in one or more of the areas and their replies were also included in our analyses.

For some of the analyses, binary variables were constructed by combining (a) those who 'Strongly Agreed/Agreed' (which we refer to as 'Agreed') versus all other responses combined and (b) those who 'Strongly Disagreed/Disagreed' ('Disagreed') versus all other responses combined. We used non-parametric tests $\left(\chi^{2}\right.$ tests, Mantel-Haenszel $\chi^{2}$ tests for a linear trend, Spearman's rank correlations) and binary logistic regression to (a) explore trends in the data; (b) assess variation between doctors who graduated from different medical schools, between men and women, and between ethnic white doctors and nonwhite doctors; and (c) compare the responses of the graduates of 2011 and 2012 with those of six previous cohorts: the qualifiers of 2008 and 2009 (and ref. 13 as published in ref. 15) and 1999, 2000, 2002 and 2005 cohorts (as published in ref. 14). The questionnaires sent to graduates of the 1999 and 2000 cohorts only contained the main question of preparedness and not the two subsidiary questions. The cohorts of 2008 and 2009 included graduates from five new medical schools not present in earlier cohorts, and the 2012 cohort included graduates from one additional new school. Since multiple analyses were performed on the same dataset, we deemed differences to be significant at $\mathrm{p} \leq 0.01$.

\section{RESULTS}

Excluding graduates who were deceased, declined to participate, were untraceable, or responded to a short version of the questionnaire that did not contain the preparedness question, the overall response rate was 45\% (2324/5171) for the 2012 cohort who were contacted by post and email, and 15\% (1000/5615) for the 2011 cohort who were contacted by email alone. The response rate for the 2012 cohort is similar to the $46 \%$ obtained for the 2008 cohort and the $44 \%$ obtained for the 2009 cohort. $^{1415}$

\section{Increased sense of preparedness over time}

More than two-thirds of respondents from both the 2011 and 2012 cohorts 'Agreed' that medical school had prepared them well for work (2011: 68\% (95\% CI 64.6\% to 70.5\%); 2012: $70 \%(68.4 \%$ to $72.2 \%)$ ). There was no statistically significant difference between the 2011 and 2012 cohorts in the percentage that 'Agreed' $\left(\chi^{2}=2.3, \mathrm{df}=1, \mathrm{p}=0.13\right)$ or 'Disagreed' $\left(\chi^{2}=4.9, \mathrm{df}=1, \mathrm{p}=0.03\right)$ that they were well prepared; however, the percentage that 'Strongly Agreed' that they were well prepared was greater in the 2012 cohort
$(24 \%)$ than in the 2011 cohort $\left(15 \% ; \chi^{2}=25.9, \quad d f=1\right.$, $\mathrm{p}<0.001)$.

There was a marked increase, over time, in self-reported preparedness for work among medical graduates from 1999 to 2012 (figure 1). Across the eight cohorts surveyed, the percentage of doctors who 'Agreed' that they were well prepared for work doubled from $35 \%$ in 1999 to $70 \%$ in $2012\left(\chi^{2}=512.5\right.$, $\mathrm{df}=1, \mathrm{p}<0.001$ ), while the percentage who 'Disagreed' with this statement declined from 43\% in 1999 to $14 \%$ in $2012\left(\chi^{2}\right.$ trend $=512.5, \mathrm{df}=1, \mathrm{p}<0.001)$. Almost a quarter of the graduates of $2012(24 \%$; 553/2308) 'Strongly Agreed' that their experiences at medical school had prepared them well for work compared with just 4\% (95/2383) of the graduates of 1999 (figure 1).

\section{Differences in preparedness by medical school, sex and ethnicity}

We compare the responses of the 2011/2012 cohorts combined with those of the 2008/2009 cohorts combined and the earlier cohorts combined. Although doctors in the 2011/2012 cohorts felt far more prepared overall than had graduates of earlier cohorts, there remained large variation between medical schools in the percentage who 'Agreed' that their medical school had prepared them well for work $\left(\chi^{2}=213.18, \mathrm{df}=27, \mathrm{p}<0.001\right.$; figure 2). Controlling for the possible confounding effects of sex and ethnicity with binary logistic regression did not diminish the significance of medical school as a predictor of the proportion of doctors who 'Agreed' that they were well prepared (Wald's statistic $=117.0, \mathrm{df}=27, \mathrm{p}<0.001$ ).

The percentage of 2011/2012 respondents who 'Agreed' that they were well prepared varied by $44 \%$ across the 29 medical schools (from 53\% to 97\%; figure 2). This was a narrower range than reported for earlier cohorts: there was a $55 \%$ difference in the 2008/2009 cohorts $^{15}$ and $52 \%$ difference for the earlier cohorts. ${ }^{14}$ Preparedness among graduates increased (compared with previous cohorts) across almost all medical schools, substantially in some, and did not decrease appreciably at any school (figure 2).

There was very strong agreement between the ranks of medical schools in the 2008/2009 and 2011/2012 cohorts (ranked by the proportion of doctors agreeing that they were well prepared and comparing with data from 15; Spearman's $r=0.65, p<0.001$ ), but weaker agreement between rankings of schools in 2011/2012 and those prior to 2008/2009 (comparing with data from 14; Spearman's $r=0.47, p=0.02$ ). The newest medical school was ranked highest among the graduates of 2011/2012 (figure 2).

Perceptions of preparedness did not differ statistically between men and women in the 2011/2012 cohorts, and differences in the earlier cohorts, though significant in the 2008/ 2009s, were modest (table 1). In the 2011/2012 cohorts, as in previous cohorts, white doctors were more likely than nonwhite doctors to 'Agree' that they felt well prepared for work, but were not more likely to 'Disagree' (table 1).

\section{Extent to which feeling unprepared was a problem}

Among the graduates of 2011/2012, lack of preparedness was scored as having been a serious problem by $1.4 \%$ of all respondents, and a medium-sized problem by a further $16.5 \%$ of respondents (table 2). These values are lower than those reported previously (table 2 ).

Merging the serious and medium-sized response categories to form a binary variable, we found that, unlike in earlier cohorts, ${ }^{14}$ there were no significant differences in this respect 


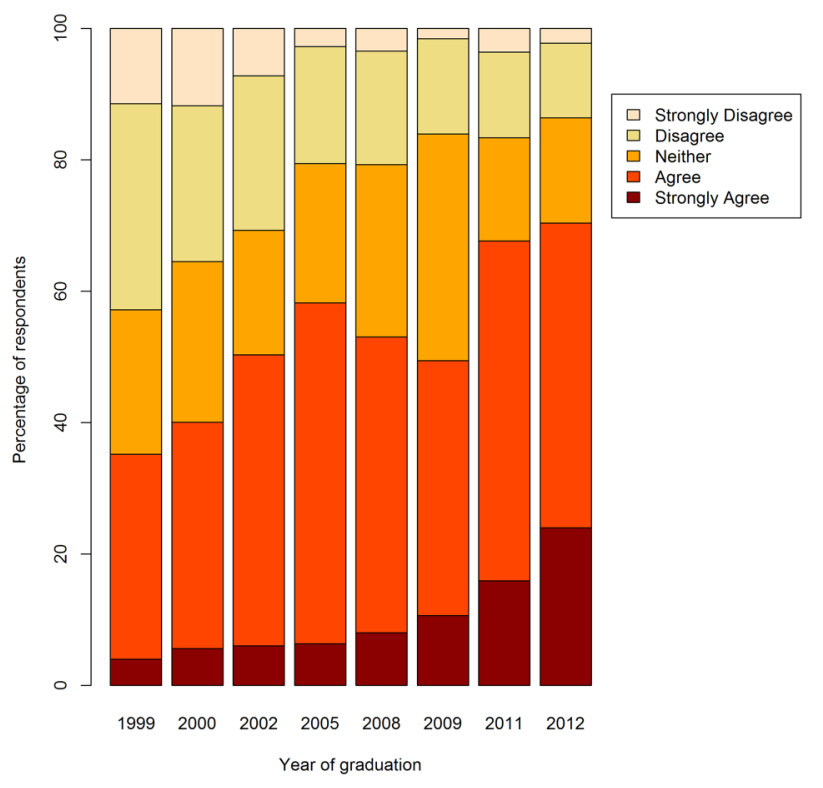

Figure 1 Percentages of doctors in each cohort responding to the statement 'My experience at medical school prepared me well for the jobs I have undertaken so far' on a five-point scale from 'Strongly Agree' to 'Strongly Disagree'.

in the responses of men and women $\left(\chi^{2}=1.46, \mathrm{df}=1\right.$, $\mathrm{p}=0.23)$, or white and non-white doctors $\left(\chi^{2}=0.04, \mathrm{df}=1\right.$, $\mathrm{p}=0.84)$.

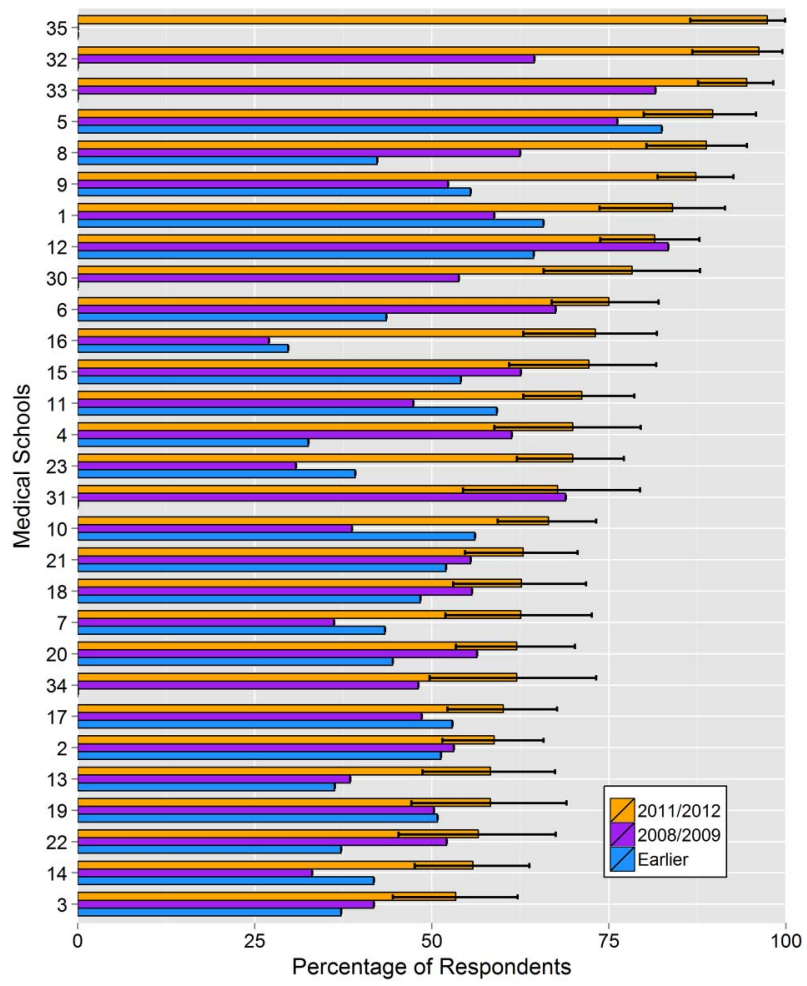

Figure 2 Percentage of first-year doctors from each UK medical school who 'Agreed' that their medical school had prepared them well for work. Medical schools are coded by numbers that correspond to the numbers used in ref. 15. Responses are shown for graduates of 2011/ 2012, 2008/2009 and earlier cohorts (1999/2000/2002/2005)

combined. Some recently formed medical schools do not appear in older cohorts. To avoid visual clutter, $95 \% \mathrm{Cls}$ are not shown for the earlier cohorts but can be found in refs. 14 and 15 .
Areas of work in which doctors felt unprepared

Doctors were also asked to indicate in which, if any, of five areas of work they felt unprepared. Results for the 2011/2012 cohorts were broadly comparable to those obtained for previous cohorts (table 2). As in previous cohorts, the area of work for which most graduates felt unprepared was 'Administrative Tasks', while few graduates felt unprepared for the 'Interpersonal Skills' required for their work (table 2). As in previous cohorts, there was considerable variation between medical schools in the percentage of the 2011/2012 graduates who indicated areas of work for which they felt unprepared (see online supplementary figure S1). Compared with graduates of 2008/ 2009 and the earlier cohorts, differences in the percentage of men and women and white and non-white doctors specifying particular areas of work as problematic were far more marginal in the 2011/2012 cohorts (table 3).

\section{DISCUSSION}

Using surveys of eight cohorts of graduate doctors spanning 13 years, we show a marked increase in self-reported preparedness for practice among recent cohorts. We found that the percentage of doctors who agreed that their medical school had prepared them well for clinical work doubled from 1999 to 2012, while the percentage who strongly agreed with this statement increased fourfold. In addition, we found that fewer graduates in the 2011/2012 cohorts considered their lack of preparedness to be a serious or medium-size problem. Our finding that $70 \%$ of the graduates of 2012 agreed that they were well prepared corresponds very closely to the GMC's recent findings into preparedness for practice $(69.9 \%$ of $\mathrm{F} 1$ graduates of 2014 agreed or strongly agreed that they were 'adequately prepared for their first Foundation post'). ${ }^{16}$

An important result from this study was that self-reported preparedness for work among the graduates of 2011/2012 increased across almost all UK medical schools. This indicates that the overall increase in preparedness of graduates we observed was a nationwide phenomenon and not just driven by a subset of graduates from a few schools. While this is an encouraging result for medical educators in the UK, we cannot confirm from this study whether this near-universal improvement is a result of ongoing changes to clinical courses, to some other aspect of undergraduate or postgraduate training, ${ }^{17}{ }^{18}$ or even to differences in the characteristics of recent graduates. ${ }^{19}$ Previous studies have reported that integrated learning programmes and those that offer greater opportunities to experience practical clinical work can facilitate the acquisition of core medical skills and knowledge by medical students. ${ }^{20-22}$ In addition, more structured induction processes, particularly those involving shadowing, have also been shown to improve clinical skills, competence and preparedness among new doctors. ${ }^{23}$ The results of our study support these previous findings by indicating that such changes to medical education and more comprehensive induction programmes in the UK are probably among the key factors in alleviating some of the apprehensions regarding preparedness for work felt by junior doctors. Our results are also consistent with other studies that have reported that recent qualifiers feel better prepared than those in the past. ${ }^{24}$

Almost all the graduates (97\%) from the top-ranked medical school, in this respect, agreed that they were well prepared for clinical work compared with just half of the graduates $(53 \%)$ from the lowest-ranked school. Given that many factors beyond curriculum and policy contribute to the learning environment, it may be unreasonable to expect 'no variation' among medical 
Table 1 Percentages of doctors who agreed, and who disagreed, that "My experience at medical school prepared me well for the jobs I have undertaken so far" by sex and ethnicitył

\begin{tabular}{|c|c|c|c|c|c|c|}
\hline & \multicolumn{6}{|c|}{ 'My experience at medical school prepared me well' } \\
\hline & \multicolumn{3}{|l|}{$\%$ Agreed } & \multicolumn{3}{|c|}{$\%$ Disagreed } \\
\hline & $2011 / 2012$ & $2008 / 2009$ & $1999 / 2000 / 2002 / 2005$ & $2011 / 2012$ & $2008 / 2009$ & $1999 / 2000 / 2002 / 2005$ \\
\hline Men & 71.4 & $54.1^{*}$ & 48.8 & 14.8 & 16.7 & 29.5 \\
\hline Women & 68.4 & 50.0 & 47.9 & 14.4 & 19.6 & 31.6 \\
\hline White & $72.4 \dagger$ & $53.6+$ & $49.3+$ & 13.1 & $18.0^{*}$ & 30.9 \\
\hline Non-white & 64.1 & 44.2 & 45.3 & 14.9 & 20.6 & 30.7 \\
\hline
\end{tabular}

schools. $^{25}$ Nevertheless, our finding that the disparity among different medical schools has lessened in the 2011/2012 cohorts compared with previous cohorts ${ }^{14} 15$ indicates that it should be possible to narrow this gap further. The percentage of UK medical graduates who reported feeling well prepared for clinical work falls short of that achieved in Denmark (83\% of graduates reported feeling prepared for work). ${ }^{26}$ This, too, indicates scope for further improvement in the UK.

We report in this study that differences in the responses by sex and ethnicity in the 2011/2012 cohorts were more marginal than those found among earlier cohorts. ${ }^{13} 14$ In contrast to our previous studies, we found no significant differences between men and women of the 2011/2012 cohorts in overall preparedness, in the extent to which lack of preparedness was a problem, or in the types of tasks that they considered to be problematic. Unlike previous cohorts, white and non-white doctors in the 2011/2012 cohorts did not differ in the extent to which they perceived lack of preparedness to be a problem, or in the types of tasks they found problematic (with the exception of physical/

Table 2 Percentages of all respondents who specified (a) that lack of preparedness was a serious, medium-sized or minor problem for them, and (b) that they felt unprepared in each area of work: average across medical schools*

\begin{tabular}{|c|c|c|c|}
\hline & \multicolumn{3}{|c|}{ Graduating cohorts } \\
\hline & $\begin{array}{l}2011 / \\
2012\end{array}$ & $\begin{array}{l}2008 / \\
2009\end{array}$ & $\begin{array}{l}1999 / 2000 / 2002 / \\
2005\end{array}$ \\
\hline \multicolumn{4}{|l|}{ Extent of problem } \\
\hline Serious & 1.4 & 2.7 & 1.7 \\
\hline Medium & 16.5 & 22.5 & 17.9 \\
\hline Minor & 29.1 & 28.1 & 20.2 \\
\hline \multicolumn{4}{|l|}{ Areas of work } \\
\hline Clinical knowledge & 10.8 & 17.4 & 11.7 \\
\hline Clinical procedures & 15.4 & 21.2 & 20.5 \\
\hline Administrative tasks & 23.9 & 31.6 & 22.0 \\
\hline Interpersonal skills & 3.0 & 2.7 & 1.5 \\
\hline $\begin{array}{l}\text { Physical/emotional/mental } \\
\text { demands }\end{array}$ & 17.4 & 26.2 & 15.8 \\
\hline \multicolumn{4}{|c|}{$\begin{array}{l}\text { Responses are shown for graduates of 2011/2012 combined, 2008/2009 combined } \\
\text { and } 1999 / 2000 / 2002 / 2005 \text { combined. } \\
\text { *The denominator in all cases is all respondents: } 3324 \text { for the graduates of } 2011 / \\
2012,5410 \text { for the graduates of } 2008 / 2009 \text { and } 9025 \text { for the earlier cohorts (data for } \\
2008 / 2009 \text { are adapted from Svirko et } a l^{13} \text { and for the earlier cohorts from Goldacre } \\
\text { et } a l^{14} \text { ). }\end{array}$} \\
\hline
\end{tabular}

emotional/mental demands; refs. 13, 14). It seems plausible that moves towards project-based, focused learning in smaller groups may have benefitted some women and ethnic minority students through the greater degree of 'inclusivity' that such teaching methods afford individual students. ${ }^{27}$ Elsewhere, we have discussed at length the implications and possible causes of differences in perceived preparedness for work comparing ethnic groups. ${ }^{13}$ The fact that differences have persisted despite greater overall preparedness among recent graduates should be investigated further.

This study covers the views of a very large number of junior doctors from all medical schools in the UK in cohorts that span a 13-year period. Our surveys are conducted independent of any organisation that employs, trains or influences the doctors' careers. We therefore believe that we get honest answers from respondents. Nevertheless, there are certain limitations to our study. We present analyses of self-reported preparedness for work among newly qualified doctors, which may or may not correlate with independent assessments of their clinical competency. ${ }^{28}$ It is possible that self-reported preparedness might reflect, at least in part, respondents' confidence (or lack of it) or self-awareness rather than actual levels of 'preparedness'. Comparing independent tests of doctors' abilities with their selfreported responses could distinguish between these possibilities and may be a fruitful area for further research.

As with all voluntary surveys, our results may be susceptible to non-respondent bias. Considering this, we compared the responses of those who replied early (to the first or second survey mailing) with those who replied late (to one of the subsequent mailings, and who would have been non-responders had we not persevered with follow-up mailings). There was no significant difference in percentages that agreed that they were prepared for work (respectively, $70.3 \%$ c.f. $68.9 \%$, as was found in our previous studies). ${ }^{13}{ }^{15}$ Hence, non-respondent bias is not likely to be a major problem. It is worth noting that we obtained a low response rate for our 'email only' survey of the graduates of 2011 and thus hard-copy post remains an essential part of our survey method.

Ensuring that graduates are well prepared for clinical practice is a key factor in facilitating a smooth transition from student to new doctor. We have shown that self-reported preparedness among recent graduates is higher than at any other time in the past 13 years. Thus, our work contributes to a growing body of evidence suggesting that recent reforms to medical education in the UK are helping to produce doctors who feel 'prepared' for the challenges of being a doctor. Focused studies of particular 
Table 3 Differences by sex and ethnicity in the percentage of doctors who felt unprepared in each of five areas of work (graduates of 2011/ 2012 combined; $\mathrm{N}=3324$ )

\begin{tabular}{|c|c|c|c|c|c|}
\hline & Clinical knowledge & Clinical procedures & Administrative tasks & Interpersonal skills & Physical/emotional/mental demands \\
\hline Men & 12.6 & $18.6^{(a, b)}$ & $33.1^{(a, b)}$ & 4.3 & $20.0^{(a, b)}$ \\
\hline Women & 15.6 & 21.2 & 28.5 & 4.2 & 24.3 \\
\hline White & 8.8 & $13.5^{(\mathrm{a})}$ & $20.1^{(\mathrm{a})}$ & $2.6^{(a, b)}$ & $13.5 t^{(a, b)}$ \\
\hline Non-white & 11.2 & 14.9 & 24.9 & 4.6 & $21.5 t$ \\
\hline
\end{tabular}

tIndicates $p<0.01$ for comparisons within table cells. Superscripts in brackets indicate that comparisons between these subgroups were statistically significant ( $p<0.01$ ) in previous cohorts: (a) the 2008/2009 cohorts $^{13}$ and (b) the earlier cohorts (ie, 1999/2000/2002/2005). ${ }^{14}{ }^{15}$

elements of learning and training could help identify methods for further improving 'preparedness' in first-year doctors. Seeking advice and input, systematically, from students, junior doctors and senior doctors may also help identify ways of improving the extent to which newly qualified doctors feel well prepared for work.

\section{Main messages}

- Recent graduates of UK medical schools feel better prepared for clinical work than did their predecessors.

- The increase in perceptions of preparedness among graduates has occurred across almost all medical schools and constitutes nationwide progress, suggesting that medical education reforms are succeeding in producing better-prepared doctors.

- Despite improvement in overall preparedness, considerable differences remain between the graduates of different medical schools.

\section{Current research questions}

- Which particular elements of learning and training can now be enhanced to further improve 'preparedness' in junior doctors?

- Will the compulsory period of 'shadowing', introduced for all medical students in 2014, improve views about preparedness among junior doctors?

- Can differences in practice be identified between medical schools whose graduates feel that they are well prepared for work and those whose graduates feel on average less well prepared?

- What are the underlying reasons for the relatively small but persistent ethnic differences in perceived preparedness?

Acknowledgements We thank Janet Justice and Alison Stockford for data entry. We are very grateful to all the doctors who participated in the surveys.

Contributors All authors had full access to all the data (including statistical reports and tables) in the study and take responsibility for the integrity of the data and the accuracy of the data analysis. MJG and TL planned and designed the surveys. SL and TL planned the data analysis. SL undertook the data analysis. SL wrote the first draft of the paper and all authors contributed to further drafts and approved the final version.

Funding This report is an independent research commissioned and funded by the Department of Health Policy Research Programme (Cohort Studies of Doctors' Careers, project number 016/0118).
Disclaimer The views expressed in this publication are those of the authors and not necessarily those of the Department of Health.

Competing interests None declared.

Ethics approval National Research Ethics Service, following referral to the Brighton and Mid-Sussex Research Ethics Committee in its role as a multicentre research ethics committee (ref 04/Q1907/48 amendment Am02 March 2015).

Provenance and peer review Not commissioned; externally peer reviewed.

Data sharing statement It may be possible for the authors to make tabulated data, produced in the course of this work but not included in the paper, available to interested readers on request. Please contact the corresponding author.

Open Access This is an Open Access article distributed in accordance with the Creative Commons Attribution Non Commercial (CC BY-NC 4.0) license, which permits others to distribute, remix, adapt, build upon this work non-commercially, and license their derivative works on different terms, provided the original work is properly cited and the use is non-commercial. See: http://creativecommons.org/ licenses/by-nc/4.0/

\section{REFERENCES}

1 Jones A, McArdle TJ, O'Neill PA. How well prepared are graduates for the role of pre-registration house officer? A comparison of the perceptions of new graduates and educational supervisors. Med Educ 2001;35:578-84.

2 Goldacre MJ, Lambert T, Evans J, et al. Preregistration house officers' views on whether their experience at medical school prepared them well for their jobs: national questionnaire survey. BMJ 2003;326:1011-12.

3 Ochsmann EB, Zier U, Drexler $\mathrm{H}$, et al. Well prepared for work? Junior doctors' self-assessment after medical education. BMC Med Educ 2011;11:99.

4 Frambach JM, Manuel BAF, Fumo AMT, et al. Students' and junior doctors' preparedness for the reality of practice in sub-Saharan Africa. Med Teach 2015;37:64-73

5 Goodyear HM. First year doctors experience of work related wellbeing and implications for educational provision. Int J Med Educ 2014;5:103-9.

6 Gunasingam N, Burns K, Edwards J, et al. Reducing stress and burnout in junior doctors: the impact of debriefing sessions. Postgrad Med J 2015;91: 182-7.

7 General Medical Council. Tomorrow's doctors. Recommendations on undergraduate medical education. Secondary Tomorrow's doctors. Recommendations on undergraduate medical education. 1993. http://www.gmc-uk.org/Tomorrow_s_ Doctors 1214.pdf 48905759.pdf

8 General Medical Council. Tomorrow's doctors. London: General Medical Council, 2003.

9 Wood D. ABC of learning and teaching in medicine: Problem based learning. BMJ 2003:326:328-30.

10 General Medical Council. Tomorrow's doctors: outcomes and standards for undergraduate medical education. London: GMC, 2009.

11 General Medical Council. Promoting excellence: standards for medical education and training. London: General Medical Council, 2015.

12 Medical Schools Council. Annual review 2014. London: Medical Schools Council, 2014.

13 Svirko E, Lambert T, Goldacre MJ. Gender, ethnicity and graduate status, and junior doctors' self-reported preparedness for clinical practice: national questionnaire surveys. J R Soc Med 2014;107:66-74.

14 Goldacre MJ, Taylor K, Lambert TW. Views of junior doctors about whether their medical school prepared them well for work: questionnaire surveys. BMC Med Educ 2010;10:78.

15 Goldacre MJ, Lambert TW, Svirko E. Foundation doctors' views on whether their medical school prepared them well for work: UK graduates of 2008 and 2009. Postgrad Med J 2014;90:63-8.

16 General Medical Council. Be prepared: are new doctors safe to practise? Manchester: General Medical Council, 2014. 
17 Evans DE, Wood DF, Roberts CM. The effect of an extended hospital induction on perceived confidence and assessed clinical skills of newly qualified pre-registration house officers. Med Educ 2004;38:998-1001.

18 Van Hamel C, Jenner LE. Prepared for practice? A national survey of UK foundation doctors and their supervisors. Med Teach 2015;37:181-8.

19 Cave J, Woolf $\mathrm{K}$, Jones $\mathrm{A}$, et al. Easing the transition from student to doctor: how can medical schools help prepare their graduates for starting work? Med Teach 2009;31:403-8.

20 Wiener-Ogilvie S, Bennison J, Smith V. General practice training environment and its impact on preparedness. Educ Prim Care 2014;25:8-17.

21 Jones $A, M c A r d l e ~ P, O^{\prime}$ Neill P. Perceptions of how well graduates are prepared for the role of pre-registration house officer: a comparison of outcomes from traditional and an integrated PBL curriculum. Med Educ 2002;36:16-25.

22 Watmough S, Garden A, Taylor D. Pre-registration house officers' views on studying under a reformed medical curriculum in the UK. Med Educ 2006;40: 893-9.
23 Blencowe NS, Van Hamel C, Bethune R, et al. 'From scared to prepared': targeted structured induction training during the transition from medical school to foundation doctor. Perspect Med Educ 2015;4:90-2.

24 Watmough S, Taylor D, Garden A. Educational supervisors evaluate the preparedness of graduates from a reformed UK curriculum to work as pre-registration house officers (PRHO): a qualitative study. Med Educ 2006:40:995-1001.

25 McManus IC. Medical school differences: beneficial diversity or harmful deviations? Qual Saf Health Care 2003:12:324-5.

26 Morcke AM, Nielsen DG, Kjeldsen IT, et al. Medical graduates feel well-prepared for clinical work. Dan Med Bull 2011;58:A4330.

27 Hocking C, Cooke S, Bowl M. 'Academic engagement' within a widening participation context-a 3D analysis. Teach Higher Educ 2007;12:721-33.

28 Davis DA, Mazmanian PE, Fordis M, et al. Accuracy of physician self-assessment compared with observed measures of competence: a systematic review. JAMA 2006;296:1094-102. 